\title{
Aminophylline shortage and current recommendations for reversal of vasodilator stress: An ASNC information statement endorsed by SCMR
}

\author{
Aiden Abidov, MD, PhD, ${ }^{\text {a }}$ Vasken Dilsizian, $M D,{ }^{b}$ Rami Doukky, $M D, M S c,{ }^{c}$ \\ W. Lane Duvall, $M D,{ }^{d}$ Christopher Dyke, $M D,{ }^{e}$ Michael D. Elliott, $M D,{ }^{f}$ \\ Fadi G. Hage, MD, ${ }^{\mathrm{g}}$ Milena J. Henzlova, $\mathrm{MD}$, PhD, ${ }^{\mathrm{h}}$ Nils P. Johnson, $M D,{ }^{\mathrm{i}}$ \\ Ronald G. Schwartz, MD, MS, ${ }^{\mathrm{j}}$ Gregory S. Thomas, $M D,{ }^{k}$ and Andrew J. Einstein, \\ $M D, P^{1}$ \\ a Wayne State University and John D. Dingell VA Medical Center, Detroit, MI \\ b University of Maryland, Baltimore, MD \\ c Cook County Health, Chicago, IL \\ d Hartford Hospital, Hartford, CT \\ e Alaska Heart and Vascular Institute, Anchorage, AK \\ f Carolinas Medical Center, Charlotte, NC \\ $\mathrm{g}$ University of Alabama at Birmingham and Birmingham VA Medical Center, Birmingham, AL \\ h Friendship Center, Sarasota, FL \\ i University of Texas Medical School, Houston, TX \\ i University of Rochester, Rochester, NY \\ k MemorialCare Heart \& Vascular Institute, University of California, Irvine, Long Beach, CA \\ I Division of Cardiology, Department of Medicine and Department of Radiology, Columbia \\ University Irving Medical Center and New York-Presbyterian Hospital, New York, NY
}

Received Nov 5, 2018; accepted Nov 19, 2018

doi: $10.1007 / \mathrm{s} 12350-018-01548-0$

Pharmacologic reversal of serious or intolerable side effects (SISEs) from vasodilator stress is an important safety and comfort measure for patients experiencing such effects. While typically performed using intravenous aminophylline, recurrent shortages of this agent have led to a greater need to limit its use and consider alternative agents. This information statement provides background and recommendations addressing indications for vasodilator reversal, timing of a reversal agent, incidence of observed SISE with vasodilator stress, clinical and logistical considerations for aminophylline-based reversal, and alternative non-aminophylline based reversal protocols.

Key Words: MPI • vasodilators $\cdot$ SPECT $\cdot$ PET $・$ MRI

Electronic supplementary material The online version of this article (https://doi.org/10.1007/s12350-018-01548-0) contains supplementary material, which is available to authorized users.

The authors of this article have provided a PowerPoint file, available for download at SpringerLink, which summarises the contents of the paper and is free for re-use at meetings and presentations. Search for the article DOI on SpringerLink.com.

This article is co-published in the journals Journal of Nuclear Cardiology and Journal of Cardiovascular Magnetic Resonance, and is available at https://doi.org/10.1007/s12350-018-01548-0 and http s://doi.org/10.1186/s12968-018-0510-7 respectively.
Drs. Abidov, Dilsizian, Doukky, Duvall, Hage, Henzlova, Johnson, Schwartz, Thomas, and Einstein served as ASNC representatives. Drs. Dyke and Elliot served as SCMR representatives.

Reprint requests: Andrew J. Einstein, MD, PhD, Division of Cardiology, Department of Medicine, and Department of Radiology, Columbia University Irving Medical Center and New York-Presbyterian Hospital, 622 West 168th Street PH 10-203, New York, NY 10032; ae2214@cumc.columbia.edu

J Nucl Cardiol 2019;26:1007-14.

$1071-3581 / \$ 34.00$

Copyright $(\subseteq 2018$ The Author(s) 


\begin{tabular}{|ll}
\hline Abbreviations \\
ACLS & $\begin{array}{l}\text { Advanced cardiac life support } \\
\text { American Society of Nuclear }\end{array}$ \\
CMR & $\begin{array}{l}\text { Cardiology } \\
\text { Cardiovascular magnetic resonance }\end{array}$ \\
IV & Intravenous \\
MPI & Myocardial perfusion imaging \\
PET & $\begin{array}{l}\text { Positron emission tomography } \\
\text { SCMR }\end{array}$ \\
Society for Cardiovascular Magnetic \\
Resonance \\
SISE & $\begin{array}{l}\text { Serious and intolerable side effects } \\
\text { SPECT }\end{array}$ \\
& $\begin{array}{l}\text { tomgle photon emission computed } \\
\text { tomoghy }\end{array}$ \\
\end{tabular}

\section{OVERVIEW}

The purpose of this document is to provide cardiac imaging specialists performing vasodilator stress testing with specific recommendations regarding options for the reversal of vasodilator stress. The timeliness of this document is associated with recurrent shortages of aminophylline, which has been used in most laboratories for reversal of serious and intolerable side effects (SISEs) from vasodilator stress.

This information serves as a summary of expert opinion on this topic developed by the American Society of Nuclear Cardiology (ASNC) and endorsed by the Society for Cardiovascular Magnetic Resonance (SCMR). This document covers general indications for reversal of SISE from vasodilator stress, the standard reversal procedure using intravenous (IV) aminophylline, and alternative options based on evidence and expert opinion for effective and safe reversal of SISE from vasodilator stress during pharmacologic stress myocardial perfusion imaging (MPI) with single photon emission computed tomography (SPECT), positron emission tomography (PET), or cardiovascular magnetic resonance (CMR).

\section{INDICATIONS FOR VASODILATOR STRESS REVERSAL}

ASNC 2016 imaging guidelines for SPECT nuclear cardiology procedures ${ }^{1}$ support using aminophylline for reversing the effects of vasodilator stress testing with adenosine, regadenoson and dipyridamole when SISE are encountered. The guidelines reflect evidence that most vasodilator stress-related adverse effects are selflimiting and do not require reversal. Serious adverse effects can be effectively aborted with IV administration of aminophylline, a xanthine derivative and a nonselective adenosine receptor antagonist, ${ }^{2}$ with the exception of seizures. IV lorazepam should be the first-line intervention for seizures and methylxanthine use is not recommended for reversal of adenosine or regadenoson effects under these circumstances because of the concern for possible lowering of the seizure threshold and potential risk of exacerbating seizures, ${ }^{3}$ based on limited evidence and the preponderance of expert opinion.

Based on the 2016 guidelines, ${ }^{1}$ indications for reversal of vasodilator stress using IV aminophylline (50-250 mg intravenously at least 1 minute after the tracer injection) include the following:

1 Severe hypotension (systolic blood pressure (BP) $\leq 80 \mathrm{mmHg}$ ) or symptomatic hypotension;

2 Development of symptomatic, persistent Mobitz II 2nd degree or complete heart block;

3 Significant cardiac arrhythmia (e.g. ventricular tachycardia);

4 Wheezing;

5 Severe chest pain associated with ST depression of $2 \mathrm{~mm}$ or greater, or ST elevation of $1 \mathrm{~mm}$ or greater;

6 Signs of poor perfusion (pallor, cyanosis, cold or clammy skin);

7 Intolerable symptoms such as nausea, vomiting, or abdominal pain.

It is important for clinicians to identify patients at risk for SISE who may need prompt reversal of vasodilator stress agent. Those at risk include patients with borderline low systolic BP $(<100 \mathrm{mmHg})$, baseline 1 st or 2 nd degree atrioventricular block, reactive airway disease, or acute coronary syndrome presentation. ${ }^{4}$ Patients with end-stage renal disease tend to have higher frequency of regadenoson-induced gastrointestinal side effects. ${ }^{5,6}$

For CMR stress with regadenoson, in addition to SISE, pharmacologic reversal may be required for a minority of examinations $(<25 \%)$ in which rest perfusion imaging is needed for artifact differentiation observed on stress imaging. In such a case, given the half-life of regadenoson and the short window between stress and rest CMR perfusion imaging, vasodilator stress reversal may be deemed necessary if there is concern for residual drug effect.

\section{TIMING OF A REVERSAL AGENT}

Few studies have examined how early following radioisotope administration a reversal agent for vasodilator stress can be administered without influencing the results of perfusion imaging. 
The majority of myocardial uptake of the radioisotope occurs within 1-2 minutes following the radioisotope injection. ${ }^{7-10}$ Thus, it is important to maintain vasodilator-induced myocardial hyperemic state during this period. However, if a very serious adverse event were to occur, the vasodilator should be reversed immediately. If vasodilator agent reversal occurs in the first minute following radioisotope injection, the "stress" perfusion could well be compromised. Therefore, following a premature reversal of vasodilator stress, if MPI is normal or myocardial ischemia is absent in a patient with high likelihood of ischemic heart disease, the possibility of a "false-negative" scan should be considered and further testing with an alternative stress agent (such as dobutamine) should be considered. For stress CMR, a reversal agent can be given once first-pass stress perfusion images have been acquired.

\section{INCIDENCE OF OBSERVED SIDE EFFECTS WITH VASODILATOR STRESS}

Based on published evidence, the vasodilator stressor agents used in current cardiology practice are safe and have a very low observed rate of serious adverse effects (Table 1). Mild, easily reversible adverse effects are frequent. Perceived shortness of breath associated with regadenoson is commonly seen and does not reflect elevated pulmonary capillary wedge pressure, but may reflect a central nervous system effect. ${ }^{11}$ Gastrointestinal side effects are overall mild/transient and are more frequently observed in patients with end-stage renal failure undergoing regadenoson stress. ${ }^{5,6}$

Table $2^{3}$ provides more detailed information regarding SISE associated with vasodilator stress agents which essentially represents a list of indications for the reversal procedure (with the exception of seizures). As can be seen from these data, the rate of occurrence of clinical situations matching the list of indications for reversing vasodilator stress is very infrequent.

Considering combined evidence of serious adverse effects of clinically utilized vasodilator agents and actual guidelines-endorsed indications for aminophylline use, a nuclear cardiology or CMR laboratory will encounter occasional cases that require vasodilator stress reversal. The rate of these cases has not been definitively assessed but it is certainly less than $10 \%$ and may be as low as $1 \%$ based on Tables 1 and 2 . With periodic shortage of aminophylline, selective use of reversal agents is a reasonable alternative to routine use and does not compromise safety or effectiveness of testing.

\section{AMINOPHYLLINE-BASED VASODILATOR STRESS REVERSAL: CLINICAL AND LOGISTICAL CONSIDERATIONS}

Despite the data demonstrating that only a fraction of vasodilator stress studies require a reversal procedure, present day cardiac imaging laboratories utilize IV aminophylline much more frequently and with high regional variability. In different clinical practices in the US, the proportion of vasodilator stress tests with aminophylline use ranges from less than $3 \%$ to $100 \%,{ }^{12}$ the latter being in an attempt to prevent any possible adverse effects of the vasodilator stress.

Randomized controlled trials demonstrate use of aminophylline is safe, well tolerated, and effective in improving overall patient satisfaction with vasodilator stress testing, the latter by reducing uncomfortable and easily treatable symptoms. ${ }^{13,14}$ In pooled analysis of two randomized but non-crossover trials including 548 patients, investigators found that perfusion did not appear to be impacted if aminophylline was administered 1.5 minutes following Tc- $99 \mathrm{~m}$ tetrofosmin injection. ${ }^{15}$ Until further studies are performed, a delay of 2 or more minutes after tracer injection is prudent if reversal is indicated. With this delay, if SISE are encountered during vasodilator stress, the reversal procedure should be performed without concern for altering imaging findings.

To date, we do not have any published evidence whether routine or "prophylactic" vasodilator stress reversal using IV aminophylline (or any other reversal agent), regardless of whether or not a patient develops an adverse reaction, is clinically beneficial. Such prophylactic use is not included in the package inserts of regadenoson, adenosine, or dipyridamole. However, it may unnecessarily complicate the performance of vasodilator stress, introduce an intervention that may affect imaging findings if administered too soon after tracer injection, and, at least hypothetically, increase risk due to exposure to an unnecessary medical agent. Accordingly, this approach is not recommended.

\section{ALTERNATIVE NON-AMINOPHYLLINE BASED VASODILATOR STRESS REVERSAL PROTOCOLS}

When aminophylline is in short supply but reversal of SISE from vasodilator stress is indicated, alternative reversal agents that have been shown to be effective in this setting should be used.

One such agent is another xanthine derivative, theophylline, which has been reported to reverse adverse symptoms of the vasodilator stressor agents safely and effectively. Since aminophylline is a salt that contains approximately $80 \%$ theophylline by weight, lower doses 


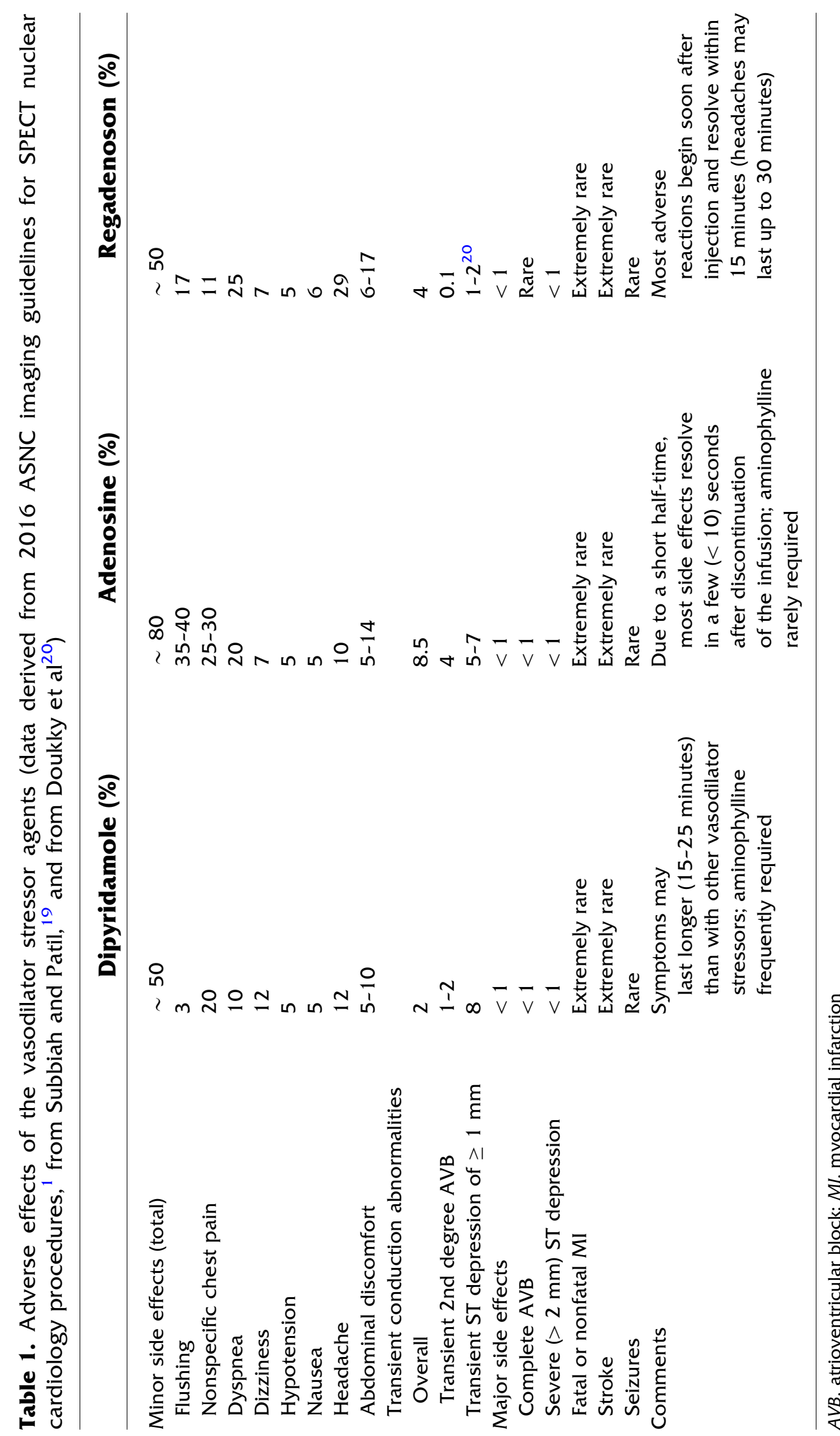




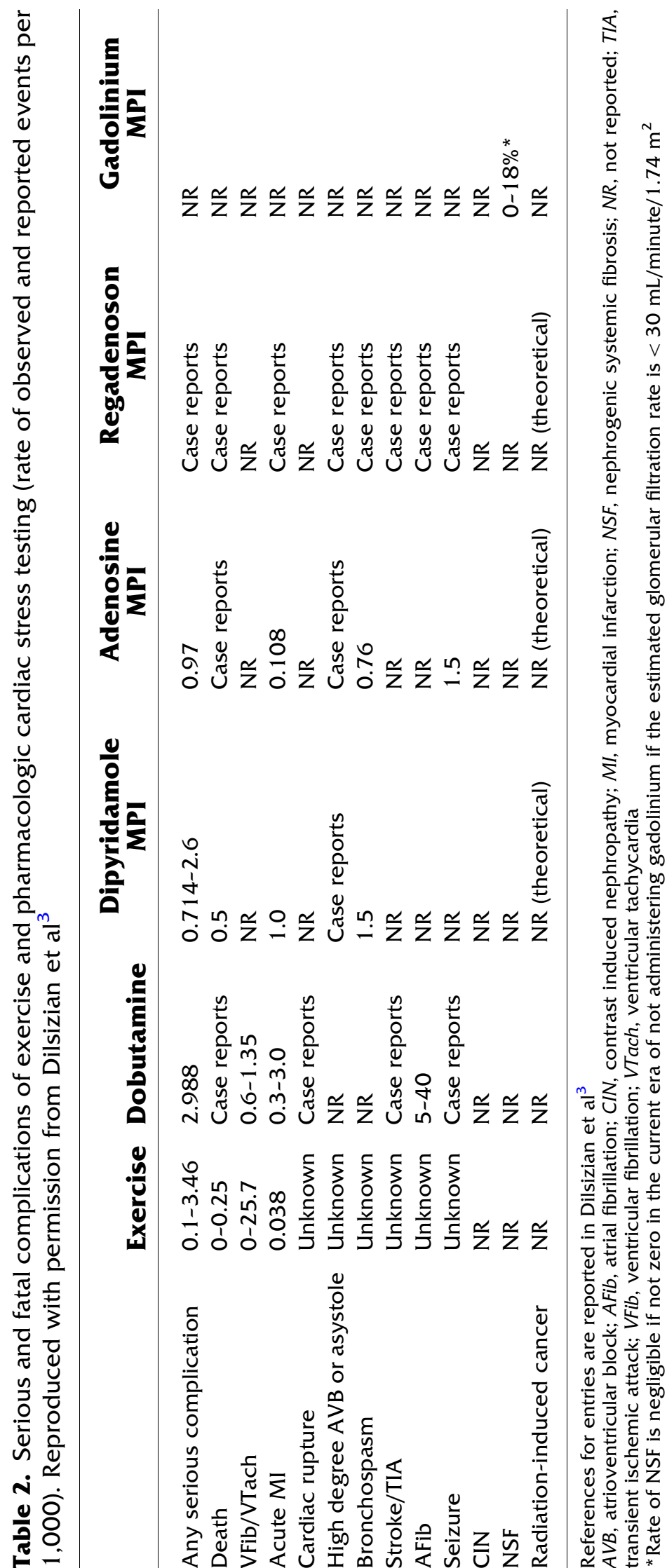




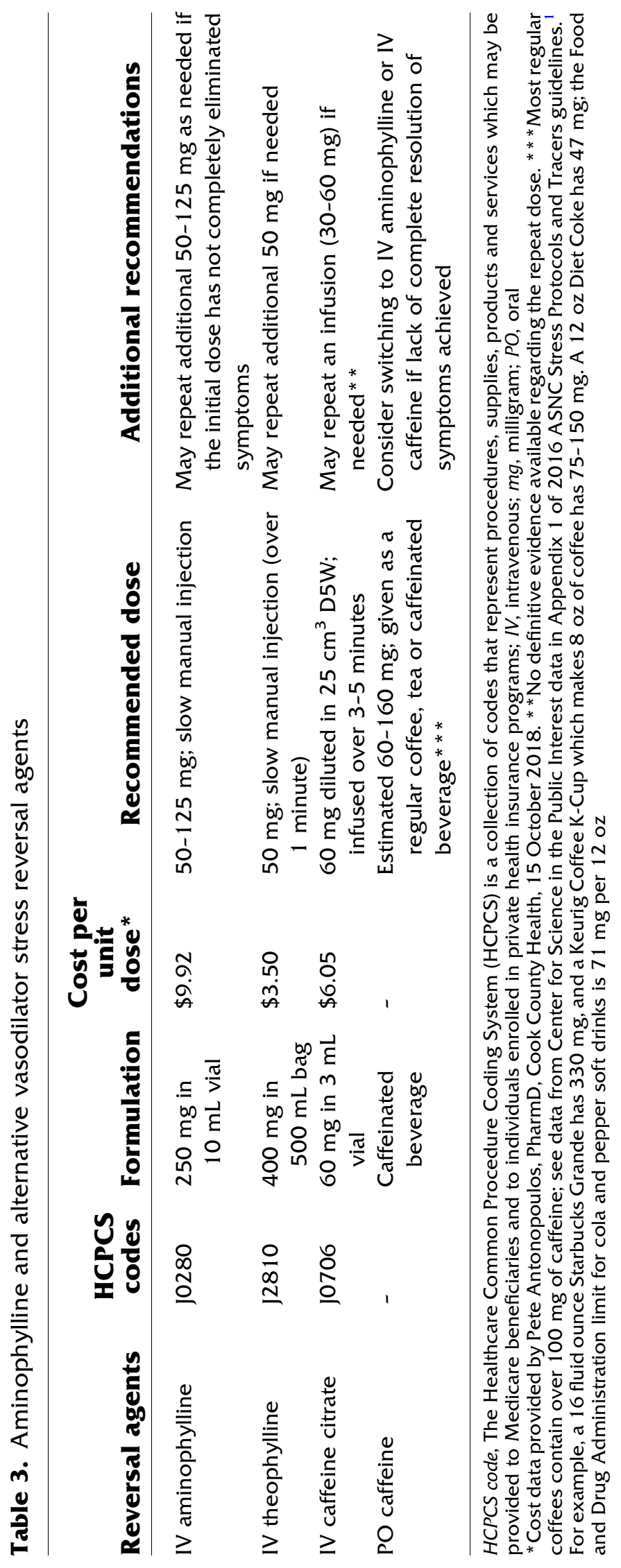


of theophylline can achieve the same reversal effects. Moreover, due to differences in concentration between aminophylline $(25 \mathrm{mg} / \mathrm{mL})$ and theophylline $(0.8 \mathrm{mg} /$ $\mathrm{mL}$ ), it becomes impractical to administer more than $50 \mathrm{mg}$ of theophylline as a single dose, equivalent to roughly $60 \mathrm{mg}$ of aminophylline. A total of 5 published series with 215 patients support the use of IV theophylline as a vasodilator stress reversal agent. ${ }^{16}$ In the largest such series, 154 patients received theophylline for reversal of dipyridamole; only 11 patients (7\%) required a second injection, and in all cases side effects or frank ischemia from dipyridamole were successfully treated. $^{16}$

Another widely available methylxanthine inhibitor of the adenosine receptors (including adenosine 2A receptors activated by regadenoson) is caffeine. This agent is administered via either an IV or oral route. Both oral and IV caffeine were recently compared in a randomized controlled fashion to IV aminophylline by Doran et $\mathrm{al}^{17}$ and were shown to provide rapid and safe reversal of vasodilator-induced adverse effects during SPECT MPI. This study evaluated the effect of reversal agents on patient-reported symptoms in a series of 241 consecutive patients presenting to the stress laboratory and undergoing regadenoson stress. Patients were randomized to IV aminophylline, to $60 \mathrm{mg}$ of IV caffeine over 3-5 minutes, or to oral caffeine as either regular coffee or diet cola taken ad lib (estimated 60-160 mg oral caffeine, with no exact dose available). No serious adverse effects of regadenoson were encountered. Of the 241 patients undergoing regadenoson stress, $152(63 \%)$ received a reversal agent. Rates of complete or predominant reversal were $100 \%$ with IV aminophylline, $96 \%$ with IV caffeine ( $P=$ NS vs aminophylline), and $84 \%$ with PO caffeine ( $P=0.003$ vs IV aminophylline). Of the 37 patients in the oral caffeine group, 19 assigned to oral caffeine crossed over to receive IV caffeine based on concern the symptoms might be too severe for oral caffeine to be effective. ${ }^{17}$

Alternative methods of caffeine administration have also been used, such as buccal administration, which is thought to be rapid and well tolerated. The only letter addressing this delivery method ${ }^{18}$ did not report rates of successful reversal of side effects or ischemic electrocardiographic changes, or the need for additional pharmacologic reversal, and thus this approach still warrants formal investigation. Direct IV injection of caffeine over a shorter period of 30-60 seconds has not been studied to date.

Data regarding use of the alternative reversal agents is summarized in Table 3. Of note, calculation of the IV or oral caffeine dose comparable with an index dose of IV aminophylline is challenging and not always feasible. $^{12}$
Finally, adding low-level exercise to vasodilator stress in patients undergoing MPI (except patients with left bundle branch block or right ventricular pacemaker), may minimize some adverse reactions during and after the vasodilator stress, but cannot completely eliminate these negative effects. ${ }^{17}$ Thus, reversal agents should be available in any stress laboratory performing vasodilator stress testing and medical staff should be familiar and efficient in recognizing SISE.

While reversal of the adverse effects is an important part of safety protocols in the modern nuclear cardiology or cardiac magnetic resonance laboratory, vasodilator induced coronary steal and ischemia in patients with coronary disease may produce angina, ischemic electrocardiographic changes and/or ischemic arrhythmias. Therefore, approved advanced cardiac life support (ACLS) protocols, in addition to reversal agent administration, must be readily available. Similar to ischemic complications, non-cardiac severe adverse reactions, such as severe wheezing, headache or seizures, require availability of a full spectrum of emergent therapeutic measures targeting these symptoms.

In the absence of comparative effectiveness studies, given the chemical similarities (aminophylline is a mixture of theophylline and ethylenediamine), the panel recommends the use of IV theophylline as a first choice reversal agent where possible when aminophylline is not available. However, shortages of these drugs have been noted in tandem, so when neither is available, IV caffeine should be used for reversal of SISE.

\section{SUMMARY}

Vasodilator stress related adverse reactions and complications may require implementation of a reversal procedure in approximately $1 \%-10 \%$ of patients. There is no definitive published evidence showing any significant clinical benefit of the preventive use of vasodilator stress reversal in patients undergoing vasodilator stress MPI who are asymptomatic. Patients should be routinely advised prior to testing that rapid, safe and effective reversal of any adverse effects of vasodilator stress testing is immediately available. Based on the available evidence, several effective and safe options for reversal of vasodilator stress-related complications/adverse reactions are available. These include IV aminophylline, theophylline, and caffeine. Theophylline and caffeine can be substituted for aminophylline in the nuclear cardiology or cardiac magnetic resonance practice in the event of a shortage of IV aminophylline. Moreover, oral caffeine is typically effective in mild cases of vasodilator stressassociated adverse reactions for most patients. These reversal agents have been shown to be safe and effective. 


\section{Authors' contribution}

Conception and design of study: AA, AJE. Drafting the manuscript: all authors. Revision of manuscript: all authors. All authors read and approved the final manuscript.

\section{Disclosures}

Aiden Abidov, MD, PhD (Writing Committee Chair): Research Grant from NIH, GE Healthcare. Vasken Dilsizian, MD: Research Grant from Siemens, GE Healthcare. Rami Doukky, MD, MSc: Research Grant from Astellas. W. Lane Duvall, MD (Advisory Board): Jubilant DraxImage, GE Healthcare. Christopher Dyke, MD: none. Michael D. Elliot, MD: none. Fadi Hage, MD: Research Grant from Astellas, GE Healthcare. Milena J. Henzlova, MD, PhD: none. Nils $P$. Johnson, MD: institution has Licensing/Consulting Agreement with Boston Scientific for the Start-Minimum FFR Algorithm. Frequently invited by academia/industry to speak at educational meetings/conferences on coronary physiology or cardiac PET and receive travel expenses and sometimes an honorarium, which I donate to my institution. Ronald G. Schwartz, MD, MS (Speakers Bureau): Astellas. Gregory S. Thomas, MD: Research Grant to institution, Amgen and Novartis (CHF Trials). Speakers Bureau, Astellas. Andrew J. Einstein, MD, PhD (Writing Committee Co-chair): Research Grants to institution from NIH, Canon Medical Systems. Consultant, GE Healthcare. Drs. Abidov, Dilsizian, Doukky, Duvall, Hage, Henzlova, Johnson, Schwartz, Thomas, and Einstein served as ASNC representatives. Drs. Dyke and Elliot served as SCMR representatives.

\section{Open Access}

This article is distributed under the terms of the Creative Commons Attribution 4.0 International License (http://creativ ecommons.org/licenses/by/4.0/), which permits unrestricted use, distribution, and reproduction in any medium, provided you give appropriate credit to the original author(s) and the source, provide a link to the Creative Commons license, and indicate if changes were made.

\section{References}

1. Henzlova MJ, Duvall WL, Einstein AJ, Travin MI, Verberne HJ. ASNC imaging guidelines for SPECT nuclear cardiology procedures: Stress, protocols, and tracers. J Nucl Cardiol 2016;23:60639.

2. Daya HA, Hage FG. Effect of aminophylline administration on the diagnostic yield of vasodilator myocardial perfusion imaging. J Nucl Cardiol 2017;24:1579-82.

3. Dilsizian V, Gewirtz H, Paivanas N, et al. Serious and potentially life threatening complications of cardiac stress testing: Physiological mechanisms and management strategies. J Nucl Cardiol 2015;22:1198-213.

4. Rai M, Ahlberg AW, Marwell J, Chaudhary W, Savino JA III, Alter EL, et al. Safety of vasodilator stress myocardial perfusion imaging in patients with elevated cardiac biomarkers. J Nucl Cardiol 2017;24:724-34.
5. Doukky R, Rangel MO, Wassouf M, Dick R, Alqaid A, Morales Demori R. The safety and tolerability of regadenoson in patients with end-stage renal disease: The first prospective evaluation. $\mathrm{J}$ Nucl Cardiol 2013;20:205-13.

6. Rangel MO, Morales Demori R, Voll ST, Wassouf M, Dick R, Doukky R. Severe chronic kidney disease as a predictor of benefit from aminophylline administration in patients undergoing regadenoson stress myocardial perfusion imaging: A substudy of the ASSUAGE and ASSUAGE-CKD trials. J Nucl Cardiol 2015;22:1008-18.

7. Sinusas AJ, Shi Q, Saltzberg MT, Vitols P, Jain D, Wackers FJ et al. Technetium-99 m-tetrofosmin to assess myocardial blood flow: experimental validation in an intact canine model of ischemia. J Nucl Med 1994;35:664-71.

8. Mousa SA, Cooney JM, Williams SJ. Relationship between regional myocardial blood flow and the distribution of ${ }^{99 \mathrm{~m}} \mathrm{Tc}$ sestamibi in the presence of total coronary artery occlusion. Am Heart J 1990;119:842-7.

9. Glover DK, Ruiz M, Yang JY, Smith WH, Watson DD, Beller GA. Myocardial ${ }^{99 \mathrm{~m}}$ Tc-tetrofosmin uptake during adenosine-induced vasodilatation with either a critical or mild coronary stenosis: Comparison with ${ }^{201} \mathrm{Tl}$ and regional myocardial blood flow. Circulation 1997;96:2332-8.

10. Watson DD, Glover DK. Overview of tracer kinetics and cellular mechanisms of uptake. In: Zaret BL, Beller GA, editors. Clinical nuclear cardiology. 4th ed. New York: Elsevier; 2010. p. 3-13.

11. Gui L, Duan W, Tian H, et al. Adenosine A2A receptor deficiency reduces striatal glutamate outflow and attenuates brain injury induced by transient focal cerebral ischemia in mice. Brain Res 2009;1297:185-93.

12. Jolly AF, Thomas GS. Intravenous caffeine: An alternative to aminophylline to reverse adverse effects during regadenoson myocardial perfusion imaging. J Nucl Cardiol 2017;24:1071-4.

13. Doukky R, Rangel MO, Dick R, Wassouf M, Alqaid A, Margeta B. Attenuation of the side effect profile of regadenoson: A randomized double-blind placebo-controlled study with aminophylline in patients undergoing myocardial perfusion imaging and have severe chronic kidney disease-The ASSUAGE-CKD trial. Int J Cardiovasc Imaging 2013;29:1029-37.

14. Doukky R, Morales Demori R, Jain S, Kiriakos R, Mwansa V, Calvin JE. Attenuation of the side effect profile of regadenoson: A randomized double-blinded placebo-controlled study with aminophylline in patients undergoing myocardial perfusion imaging. “The ASSUAGE trial”. J Nucl Cardiol 2012;19:448-57.

15. Fughhi I, Campagnoli T, Ali A, Doukky R. Impact of a regimented aminophylline administration protocol on the burden of regadenoson-induced ischemia detected by SPECT myocardial perfusion imaging. J Nucl Cardiol 2017;24:1571-8.

16. Johnson NP, Lance Gould K. Dipyridamole reversal using theophylline during aminophylline shortage. J Nucl Cardiol 2011;18:1115.

17. Doran JA, Sajjad W, Schneider MD, Gupta R, Mackin ML, Schwartz RG. Aminophylline and caffeine for reversal of adverse symptoms associated with regadenoson SPECT MPI. J Nucl Cardiol 2017;24:1062-70.

18. Matangi M, Dutchak P. Buccal caffeine for the routine reversal of Persantine. J Nucl Cardiol 2014;21:1039.

19. Subbiah R, Patil PV. Arrhythmias in vasodilator stress testing. J Nucl Cardiol 2017;24:410-2.

20. Doukky R, Nigatu A, Khan R, et al. Prognostic significance of ischemic electrocardiographic changes with regadenoson stress myocardial perfusion imaging. J Nucl Cardiol 2018. https://doi. org/10.1007/s12350-018-1415-4. 